\title{
Tangence
}

\section{Postures scripturaires, impostures identitaires}

\section{Robert Yergeau}

Numéro 56, décembre 1997

Postures scripturaires dans la littérature franco-ontarienne

URI : https://id.erudit.org/iderudit/025955ar

DOI : https://doi.org/10.7202/025955ar

Aller au sommaire du numéro

Éditeur(s)

Tangence

ISSN

0226-9554 (imprimé)

1710-0305 (numérique)

Découvrir la revue

Citer cet article

Yergeau, R. (1997). Postures scripturaires, impostures identitaires. Tangence, (56), 9-25. https://doi.org/10.7202/025955ar d'utilisation que vous pouvez consulter en ligne.

https://apropos.erudit.org/fr/usagers/politique-dutilisation/ 


\title{
Postures scripturaires, impostures identitaires
}

\author{
Robert Yergeau
}

\section{Préliminaire}

Le texte de Lucie Hotte et de François Ouellet qui accompagnait l'a appel d'articles * pour ce numéro de Tangence posait les paramètres dialectiques à l'intérieur desquels ils désiraient situer la problématique de -L'écrivain, l'écriture et la langue dans la littérature franco-ontarienne:

La représentation de l'écrivain dans le texte peut éclairer le statut social de l'écrivain, les mythes qui l'entourent, les courants esthétiques prédominants ou marginaux (enjeux thématique, sociologique, psychanalytique, etc.), elle permet également de mettre en scène l'écriture et ses diverses fonctions (esthétique, politique, cathartique, etc.) et soulève tout un questionnement sur la langue en tant que matériau sémiotique. ${ }^{1}$

Même si certaines pistes entrouvertes par cette taxinomie autorisent à emprunter cette voie, j'y constate malgré tout l'absence de la dimension identitaire. Pourtant, l'enjeu sociologique et les diverses fonctions liées à l'écriture eussent pu l'appeler - ou, à tout le moins, la suggérer. Il ne s'agit pas, certes, de souscrire bêtement à un effet de mode consécutif à un surtexte qui marque les champs de la fiction et de la réflexion au Québec depuis quelques années $^{2}$. Ni de chercher à perpétuer le poncif à l'effet que les littératures dites minoritaires, nommément la littérature franco-ontarienne, ne puissent être étudiées que dans cette

1 Lucie Hotte et François Ouellet, * Appel d'articles. L'écrivain, l'écriture et la langue dans la littérature franco-ontarienne,$s$. p.

2 Citons les livres suivants: Marcos Ancelovici et Francis Dupuis-Déri, L'archtpel identitaire, recueils d'entretiens sur l'identité culturelle, préface de Georges Leroux, Montréal, Boréal, 1997 ; Joël Des Rosiers, Théories Caraibes. Poétique du déracinement, Montréal, 'Triptyque, 1996; Simon Harel, Le voleur de parcours. Identité et cosmopolitisme dans la littérature québécotse contemporaine, Longueuil, Le Préambule, 1989; Jocelyn Létoumeau (dir.) avec la collaboration de Roger Bernard, La question identitatre au Canada francopbone. Récits, parcours, enjeux, bors-lieux, Sainte-Foy, Presses de l'Université Laval, 1994; François Paré, Les littératures de l'extguité, Ottawa, Le Nordir, 1992 ; Id., Théories de la fragilité, Ottawa, Le Nordir, 1994. 
perspective. Si j'évoque le surtexte identitaire en effervescence, qui peut aisément se transformer en brandon de discorde ${ }^{3}$, c'est dans le but de l'insérer dans une démarche réflexive constituée de deux pôles: postures scripturaires et impostures identitaires. Postures scripturaires, dis-je, car écrire c'est assumer, sinon revendiquer une a attitude particulière du corps* textuel. Quelles postures proposent Le dernier des Franco-Ontariens de Pierre Albert, French Town de Michel Ouellette et Grand ciel bleu par ici de Robert Dickson ${ }^{4}$ ? M'intéressent au premier plan les partis pris esthétiques qui fondent ces livres. Parcours anisotropes, mises en abyme/mises en procès, trous de mémoire, miroirs déformants, les postures scripturaires s'approprient les identités, les décentrent, les recentrent; seules comptent alors les marques identitaires sur les corps d'écriture comme espace tensionnel porteur d'identités esthétiques. Cependant, il serait illusoire de chercher à faire l'impasse sur les corps prescriptifs institutionnels qui façonnent à leur convenance les corps textuels. Ainsi, est-il possible de saisir l'imposture identitaire en une double perspective: d'une part, comme je viens de le mentionner, les postures scripturaires se jouent des identités, les insérent dans une entreprise esthétique qui les transforme. D'autre part, les corps prescriptifs institutionnels tirent à eux les postures scripturaires, les inféodent à un surtexte culturel et social. Entre les deux se jouent d'incessants va-et-vient dialectiques - évoquons l'image d'un sas pour définir cet "entre deux", sas qui régularise, qui valide/invalide les jeux et enjeux textuels et institutionnels.

Les quelques réflexions que je propose peuvent s'inscrire dans le prolongement du colloque tenu en mai 1996 à l'Univer-

3 La polémique récente au sujet de la publication de L'arpenteur et le navigateur par Monique LaRue le confirme. Voir à ce propos les articles de Pierre Nepveu ("Lire à l'envers *, Le Dezoir, 26-27 avril 1997, p. A11), de Lise Bissonnette (= Un cauchemar *, Le Devotr, 26-27 avril 1997, p. B3), de Micheline de Sève (.Malaise au pluriel., Le Devotr, 5 mai 1997, p. A7) et de Jeanne Demers (: Une lecture erronée et destructrice , Le Dewoir, 17-18 mai 1997, p. A9).

4 Pierre Albert, Le dernier des Franco-Ontariens, Sudbury, Prise de parole, 1992; Michel Ouellette, French Town, Ottawa, Le Nordir, 1996; Robert Dickson, Grand ciel bleu par ici, Sudbury, Prise de parole, 1997. (L'on s'étonnera peut-être de voir une pièce de théâtre à côté de recueils de poèmes. Je considère les personnages de Pierre-Paul et de Cindy au même titre que les narrateurs des recueils de poèmes: ils déploient des stratégies formelles qui mettent en scène avant tout des corps verbaux.) Enfin, je souligne qu'en tant que directeur du Nordir, j'ai publié French Toun. 
sité McGill sur a La littérature franco-ontarienne: enjeux esthétiques. Dans l'a avant-propos * à la publication des actes de ce colloque, François Ouellet disait son fait au vecteur identitaire comme parcours dialectique obligé :

Il nous semblait donc que la façon la plus sûre de faire accéder (timidement sans doute, mais il faut bien un commencement) la littérature franco-ontarienne au rang des discours du savoir était d'évacuer toute référence à la dimension identitaire dans laquelle les ouvres ont été produites, donc de lire les œuvres pour leurs qualités proprement formelles, ou si l'on préfère, selon le projet esthétique plus ou moins avoué des auteurs, qui sont autre chose que des sensibilitês à refléter la réalité. 5

François Paré, invité à prononcer la communication inaugurale, reprit la balle au bond:

La question de l'identitaire a-t-elle fait son temps? La réponse est certes non, dans la mesure où elle n'est pas résolue - pas pour moi du moins - et où elle continue de hanter la conscience collective. Mais, du même souffle, il convient d'aller plus loin. Les enjeux esthétiques qui animent toute œuvre de littérature doivent-ils nécessairement provoquer un éclatement de la communalité, mener à une irrecevabilité du nous? N'y a-t-il de rupture esthétique que dans la singularité d'un sujet, posant radicalement sa différence sur le monde? ${ }^{6}$

Sur le terrain épistémologique de l'esthétisme et de la communalité, toute entreprise esthétique peut contribuer à façonner une communalité plurivoque, hétérogène, kaléidoscopique, éclatée. Ainsi, procédons à un renversement des perspectives et considérons l'éclatement qu'évoque Paré comme un événement baroque et jubilatoire. La communalité n'a pas à se sentir menacée par quelque représentation fictionnelle que ce soit qui l'apostropherait et l'invectiverait, l'ignorerait avec superbe ou la tournerait en dérision; la communalité sera riche de toutes les entreprises de transgression, de tous les désirs et délires qui la feront/déferont, formeront/déformeront. En outre, doit-on s'interroger en termes de recevabilité et d'irrecevabilité du nous? Le nous sera toujours

5 François Ouellet, "Avant-propos :, dans Lucie Hotte et François Ouellet (dir.), La littérature franco-ontarienne: enjetux estbétiques, Ottawa, le Nordir, 1996, p. 8.

6 François Paré, "Pour rompre le discours fondateur: la littérature et la détresse", La littérature franco-ontarienne: enjeux estbétiques, op. cit., p. 18. 
recevable; le nous devrait toujours être ce que sont les sujets dans la singularité même de leur rupture esthétique.

Cela étant, l'ère du soupçon identitaire que nous traversons acquiert, dans chaque culture, des résonances contrastées. Dans cette perspective, méfions-nous de la pavlovienne envie de discréditer sans discernement toute démarche identitaire en n'y voyant que repliement frileux, ostracisme ou, pire encore, xénophobie. En soutenant qu'aux postures scripturaires correspondent des impostures identitaires - une assertion que certains jugeront au mieux éristique, au pire futile! —, je ne signifie pas évidemment que toute quête identitaire est une imposture!

\section{Le dernier des Franco-Ontariens : refus et... refus}

ô ce grand besoin d'être lu, d'être cru... (p. 91)

Je pourrais multiplier les formules antithétiques qu'autorise la lecture du Dernier des Franco-Ontariens: l'intellectuel de l'antiintellectualisme; enfermement de l'ouverture; la continuité de la rupture; la préciosité de la vulgarité; l'académisme scatologique; le décentrement du recentrement; le lieu du non-lieu; l'enracinement du déracinement; l'illusion de la désillusion, etc. Toutes ces formules qui valent ce qu'elles valent trouveraient leur point d'ancrage, leur creuset dialectique dans la sursacralisation désacralisante de la figure emblématique et propitiatoire de l'écrivain - le salut et la damnation du narrateur passant par l'écriture qui est sa (dé)raison d'être, son point de désancrage. * [É]crivons de la vraie littérature/critiquons de la vraie littérature. (p. 77), lance insolemment le narrateur dans un des nombreux paradoxes qu'il cultive pour exhiber sur l'étal de la mauvaise conscience francoontarienne son écartèlement entre être ou ne pas être en écrivant ou en n'écrivant pas. L'énonciateur du Dernier des FrancoOntariens tire la langue maternelle dénaturée à tout ce qui représente la Loi (écrivain, critique, intellectuel, professeur). Paradoxalement, il s'institue juge et juré, procureur et avocat de la défense, témoin et accusé dans ce procès sans cause ou de toutes les causes; il est (sera) ce qu'il dénonce, ce qu'il ridiculise, ce qu'il saccage.

En utilisant comme arme ce qu'il considère de la *fausse littérature - (pour discréditer, procédé antinomique oblige, la *vraie littérature *), il fera (à sa langue défendante?) de la "vraie littérature". 
Certes, le constat est simpliste: Pierre Albert ferait de la littérature sans le savoir! Mais allons un peu plus loin: le saccage de la littérature auquel il s'adonne, et qui passe par le déni forcené de toutes les formes institutionnalisées de l'écriture, leur rejet, leur mise au ban, constitue son être dans l'écriture, partant, son être au monde. Le dernier des Franco-Ontariens déroule ses fautes d'orthographe, ses barbarismes, ses solécismes, ses anglicismes, entremêle les niveaux de langue comme autant d'attentats à la pudeur langagière. Cocteau disait que la poésie est un attentat à la pudeur dans un monde d'aveugles. Pour Albert, la littérature est un attentat à la pudeur en plein jour, dans un monde sursaturé de signes qui ont perdu toute marque distinctive. Albert se drape dans une tunique du pauvre, de "mauvais goût - L Le dernier des FrancoOntariens nous contraint sans cesse à dialectiser le "bon " et le "mauvais" goût, entendu que le goût étant *presque toujours le dégoût du goût des autres ${ }^{7}$.

Procédons à un repérage. :[L]e dernier des franco-ontariens/ a débuté l'écriture d'un livre/il ne sait pas où il s'en va encore. (p. 18). Peu importe qu'il ne le sache pas. Mieux : son salut réside dans cette errance. Ne jamais demander son chemin à qui ne sait pas s'égarer, disait Roland Giguère. Le dernier des FrancoOntariens eût pu faire sien cet aphorisme. De toute façon, seul importe véritablement le chemin de l'écriture. Même si en cours de route, en cours d'écriture, il discréditera toutes représentations réelles et symboliques de l'écrivain et de la littérature. Tenez ceci : «à quoi servent donc ces écritures, ces soi-disantes [sic] poésies" [p. 13] ; "il ["l'écrivain "] finit par mourir au bout de sa peine/ au bout de son encre/il mourut incompris/et/l'on n'entendrajamais parler de lui • [p. 13]. L'écriture ne sert à rien; la poésie se voit instituer un procès d'insignifiance. L'écriture ne sert à rien, certes - sauf à l'essentiel: exister par l'écriture, éprouver son être au monde (franco-ontarien) par une entreprise de rejet, de refus qui se répand larve et boue sur tous les poèmes (sauf sur le dernier; j'y reviendrai): "que du théâtre de pauvre/de la poésie qui n'en est pas / des écritures de bas-étage vous comprenez" (p. 22). Plus Albert renie l'écriture, plus il multiplie les fautes de (dé)goût à son endroit, plus elle lui devient essentielle. D'ailleurs, que peut-on entendre dans ces vers: - [U]n jour, je n'essaierai

7 Pière Bourdicu, - Mais qui a créé les créateurs?", Questions de soctologie, Paris, Minuit, 1984. 
même pas d'écrire/un jour, je ne sauverai pas ma peau * (p. 29), si ce n'est que l'écriture peut lui sauver la peau? Triviale constatation qui s'actualise sous les coups de butoir textuels du narrateur qui chemine nolens, volens vers les deux poèmes clausulaires, creuset où se confrontent la "fausse littérature et la *vraie littérature", comme si le poète, après avoir décrié, ridiculisé, roué de coups la littérature, la jugeait digne de son combat, comme s'il mourait à la "fausse littérature" pour naître à la "vraie littérature" - sans s'apercevoir que celle-ci était peut-être celle-là et vice versa. Lisons, dans l'avant-dernier poème du recueil, "ébauche d'une poésie franco-ontarienne ", la chronique accélérée d'une mort annoncée où, dans un emballement hallucinant, mots et mort(s), écriture et existence s'apostrophent, s'affrontent dans un ultime duel où l'on assiste au baroud du dernier des FrancoOntariens: "il ne comptera pas que son texte (s'il devient jamais un/livre) soit bon, enfin qu'on le taxe de mauvais ou de bon/peu lui importe.../le dernier des franco-ontariens sait qu'il n'a pas rien à/prouver à personne - (p. 87) - sauf à lui. Il continue encore et toujours d'écrire pour (se) prouver qu'il existe en croyant ne pas exister collectivement, pour (se) prouver qu'il peut aller au bout de toutes les désillusions: "le dernier des franco-ontariens/n'a vraiment pas été un prophète dans son pays/il n'avait tout simplement pas de pays..." (p. 94). Ce voyage eût pu s'arrêter là, sur cette brèche, sur cette béance, sur ces trois points de suspension, comme une ultime invective. Succédera toutefois à cet antipanégyrique, à cet anti-Tombeau du poète franco-ontarien inconnu, à ce guet-apens dans lequel Le dernier des FrancoOntariens s'est attiré lui-même, le dernier poème du recueil, "quivive! (le dernier poème franco-ontarien). - en fait, le premier poème où le narrateur ne calomnie plus la poésie. Au début du non-livre, le narrateur se moquait ainsi de la poésie:

et le poète sortit sa plume :

les immensités silencieuses

ne sont que compagnie

des solitudes pernicieuses

que les couleurs des nuits

projettent dans l'imagination

[...] (p. 26)

Ce * mauvais * poème finissait par un tonitruant *ostie, change de record chrisse de tapette!* (p. 26). Nous étions de plain-pied dans la non-poésie du non-livre. De ce refus naîtra un autre refus, inversé cette fois, le refus d'oublier: 


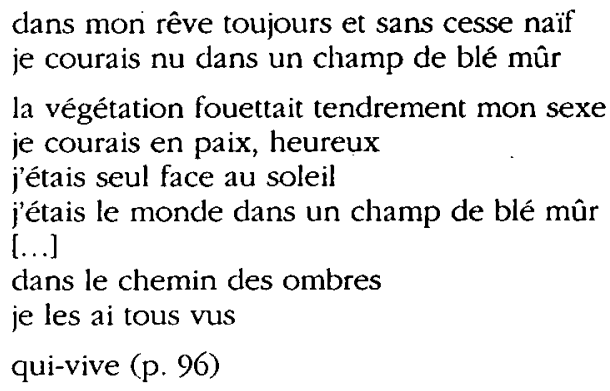

Du refus vulgaire de la poésie au refus d'oublier, le narrateur a conquis - en rêve! - tous les espaces: textuel/sexuel, réel/ rêvé, individuel/collectif. La croisade iconoclaste s'achève en épiphanie.

Venons-en aux postures scripturaires et aux impostures identitaires. J'ai délibérément choisi un livre où les marques identitaires pullulent - où le nihilisme sur l'humaine condition francoontarienne est exposé au soleil dans une sorte d'autodérision, d'autoflagellation jubilatoire. Malgré ce martèlement obsessif, cette litanie accablante, je maintiens que les postures scripturaires délivrent de faux passeports, faussent les pistes identitaires. L'identité n'est jamais là où l'on croit la débusquer. Chaque scripteur la vampirise, l'invente, la travestit, la métamorphose, l'atomise, l'énuclée, par l'entremise d'un corps de prescriptions textuelles toujours en retard ou en avance d'identité. Les écrivains concourent à un incessant trafic de petites identités. Voleurs d'identité, ils machinent l'unique et le multiple, le même et l'autre dans un transfert d'altérité. Révoquant en doute aussi bien l'universalisme (que je qualifie de) déliquescent et la communalité (illusoirement) rédemptrice, l'écrivain, quel que soit son lieu (ou son non-lieu) d'appartenance, ne devrait avoir de cesse qu'il ne mine la langue pour la faire exploser en de multiples fragments d'existence. Dans Le dernier des Franco-Ontariens, malgré les marques identitaires pléthoriques, seules (m')importent les postures scripturaires qui les subsument. L'affirmer ce n'est pas «défigurer " le recueil de Pierre Albert - en fait, c'est prendre acte de la *défiguration " à laquelle il s'est lui-même livré; défiguration qui se transforme même en une carnavalesque poétique de l'identité. L'affirmer c'est faire accéder l'écriture à elle-même. L'affirmer, enfin, c'est rappeler que la littérature doit se (dé)jouer de tout, y compris, avant tout, d'elle-même. 


\section{French Town: le surpaysement dépaysant}

"Tenté de trouver sa langue. * (p. 117)

Tirée de la scène clausulaire de la pièce de théâtre de Michel Ouellette, French Town, cette réplique de Pierre-Paul atteste l'accomplissement du drame, l'acmé des relations entre lui et son père. Pierre-Paul vient de se suicider en utilisant le fusil du père symbole de tout ce qui séparait le fils et le père (à une lettre près, - fils est l'anagramme de *fusil *; constatation facile, certes, si ce n'est que le fusil est consubstantiel au fils). Dans une prosopopée poignante, Pierre-Paul refait les gestes du père jusqu'à reparler sa langue, lui dont la langue châtiée et la répétition mécanique, obsessionnelle des règles de grammaire (sorte de psittacisme grammatical) constituaient, croyait-il, le plus sûr moyen de l'éloigner du père. Entendez toute la réplique: "Tabarnak de câlice d'ostie de chrisse, ciboire! Tenté de trouver sa langue. "Icitte câlice. Arrive icitte, toé. Chrisse, pas peur. J'te mangerai pas, stie. T'as à pleurer de même? Pleure pas de même, sacre. Pas une fille, stie." " (p. 117) Passent dans ces mots tout le sang et le sel de la pièce: la violence et le rejet, l'incompréhension et la rage, les sacres et le sacré, la puissance et l'impuissance. Quel sens donner au suicide du fils? Est-ce l'ultime geste pour ne plus entendre la langue paternelle (en se tuant, il tue la langue du père, celle qu'il a cherché - en vain - à déparler, à désapprendre)? Est-ce l'ultime geste de symbiose avec le père (cette tentative désespérée de mimétisme langagier mettait fin à son mutisme exacerbé - en parlant une *autre langue ${ }^{n}$, il s'était condamné à ne plus être compris)?

Dans French Town, le drame ne transite pas par la langue; la langue est le drame, le huis clos de la pièce, son entreprise cathartique, son exorcisme individuel et familial, son tonneau des Danaïdes, son rocher de Sisyphe. Voyez l'incipit : :Simone: Je m'appelle Simone Bédard/Pierre-Paul: Tais-toi/Cindy: Farme-toé/ Martin: Raconte. (p. 13) La mère décédée constitue la mémoire de Frencb Town, sa présence tutélaire, fantomatique - morte, elle peut parler; vivante, elle ne le pouvait pas (contrainte par les autres à se taire: - Parle pas * (p. 66), se disait-elle elle-même). Pierre-Paul et Cindy ne veulent pas entendre parler du passé: le - Farme-toé " tranchant de celle-ci la situe immédiatement dans son parti pris langagier et l'oppose violemment au "Tais-toi * altier (en apparence plus neutre, mais tout aussi violent dans sa rupture avec la langue du père et de la fille) de celui-là. En outre, le dysfonctionnement communicationnel entre Cindy et Pierre-Paul 
peut se ramener, dans les moments d'extrême tension, à l'écholalie de celui-ci et à la coprolalie de celle-là. Seul Martin veut entendre la parole maternelle, veut activer le processus anamnestique qu'il considère salutaire.

Entre le "Tais-toi " initial et la réplique - lá supplique - de la fin, le drame s'est joué, la traversée du langage a eu lieu: parole, sacre, mutisme, silence, violence verbale, vulgarité. Arrêtons-nous sur cette traversée. Dès le début, la langue fait écran entre Pierre-Paul et sa famille: "Y parle tellement bien que des fois j'ai de la misére [sic] à comprendre ce qu'y dit. [...] Un vrai dictionnaire." (p. 16) De même, présentant sa fille, elle dit : "A sacre tout le temps. (p. 17); "A parle comme son pére [sic] Gilbert. A va finir comme lui..." (p. 17) Notons le renversement des rôles: Cindy ne finira pas comme son père, alors que PierrePaul, qui refuse de parler comme son père, finira par se tuer par faire sienne la violence paternelle.

Inlassablement, Pierre-Paul égrène le chapelet des règles de grammaire. Fascinante incantation! Délirante psalmodie! Simone dit encore: "Pis Gilbert y en a fait un gars [de Cindy], parce son premier fils [Pierre-Paul] voulait pas être comme lui." (p. 21) Il ne voulait tellement pas être comme lui qu'il n'aura de cesse à la fin de copier ses gestes, de revêtir sa "combinaison de mécanicien * (p. 116) transformée en linceuil. Mais Pierre-Paul n'en est pas encore là. Le voici se réfugiant dans sa chambre, s'évadant dans le dictionnaire, cette cathédrale de mots : "Et je prenais mon Petit Larousse illustré, le seul livre dans cette maison d'illettrés. Et pendant des heures je tournais les pages. J'apprivoisais les mots. Je les découvrais. Quelle chaleur! Quel réconfort!•(p. 34) Les mots acquièrent un pouvoir transcendant; le dictionnaire est vénéré comme une bible (au sens étymologique de *livres saints "; et, si je puis me permettre, de livre qui ceint Pierre-Paul de son aura). Pierre-Paul reprend vie chaque fois qu'il trouve refuge dans le dictionnaire, ce sésame libérateur:

Je ne voulais pas sortir. Je cherchais un vocabulaire pour dire ma peine. Je me sauvais dans les pages de ce dictionnaire. Je goûtais, enfin, à la liberté. [...] Bientôt, mon âme se calmait. J'allais au début du dictionnaire. Je mémorisais les règles de grammaire, les tableaux de conjugaison, le pluriel des noms, les préfixes et les suffixes. Quelle joie! (p. 35)

L'entéléchie par le pouvoir palingénésique de la grammaire! Pour oublier la loi du Père, d'autres lois. Celle-là se manifestera avec 
18

cruauté lors de la scène de chasse initiatique - qui préfigure la scène finale - à laquelle est contraint de participer Pierre-Paul. Son père l'oblige à prendre le fusil; il ressent un malaise; il tire précipitamment; "la balle fracasse la fenêtre arrière de la camionnette" (p. 42). Son père, fou de rage, le bat. À la fin de la pièce, Pierre-Paul ne manquera pas son coup; il se tirera une balle dans la tête; son père aurait-il été fier de lui?

Un mot encore de l'initiation avortée de Pierre-Paul. Hébété, il voulait tuer son père. Violence, mort et grammaire se lient, s'enchevêtrent: "Le conditionnel exprime une action qui dépend, dans sa réalisation, de certaines conditions... J'aurais dû lui tirer dans le dos... Conditionnel passé... Une action qui n'a pas pu se réaliser. "(p. 46) Citons cette autre scène où le père bat son fils: "Mais, lui, il frappait et frappait et frappait. Il voulait m'écraser dans le plancher. (pause) Tout ça parce qu'il n'avait pas compris le verbe revendiquer... Cette nuit-là, j'ai fui. * (p. 77) Comment ne pas être interdit devant une telle fureur paternelle causée par la vertigineuse incompréhension d'un verbe! De même, comment ne pas être stupéfait devant l'exécration qu'entraînent les agrands mots" de Pierre-Paul: "Pas avec tes grands mots que tu vas m'arrêter quand je vas t'être décidée de te mettre du plomb dans le corps, toé."( $\mathrm{p}$. 64) Plus tard, Pierre-Paul mènera lui-même à terme ces propos comminatoires; il aura échoué à rompre le cercle familial (sa violence verbale et physique), au point où il s'en échappera - ou le réintégrera - par la violence.

Mettons en relief deux autres scènes. La première porte sur l'obsession de Pierre-Paul qui veut vendre à tout prix la maison familiale, pour liquider le passé. Exaspérée par Pierre-Paul qui veut la chasser de la maison car elle fait fuir les acheteurs potentiels, Cindy lui répond avec la langue du père, la langue du fusil, la seule qu'elle connaisse pour empêcher son frère de parler, de l'agresser en parlant une langue qui la dépossède:

T'ai dit de la fermer. Farme! Sinon va avoir du sang partout icitte. Ton sang. Ton estie de sang de cochon... Hein, te l'ai fermée, la yeule, moé! Hein! (elle lui passe le bout du fusil sur les lèvres) Qu'est-ce ça te fait? Comme donner un bec à la mort, hein? Donnes-y un beau bec, là. Chrisse, pourrais tirer là pis tu la ravalerais, ta maudite belle langue de cul! (p. 67)

Tout se télescope jusqu'à l'incandescence dans cette réplique: langue, violence, domination de Cindy (qui poursuit celle 
du père), le fusil comme symbole phallique - ce qui pourrait aisément conférer un autre sens à la scène de chasse, et à celle où Pierre-Paul est incapable d'avoir des relations sexuelles (ne parlant plus la langue du père, n'ayant pas accepté sa violence, il serait devenu impuisant). Enfin, Martin, le cadet, se trompe lorsqu'il essaie de calmer les esprits en disant que Pierre-Paul et Cindy se parlent sans s'écouter (p. 72). Ils ne parlent tout simplement pas la même langue.

Une dernière scène annonce la scène finale du suicide: celle où Martin apprend à Pierre-Paul qu'il ne retournera pas à l'université. Il venait de se rendre compte qu'il étudiait juste pour faire plaisir à Pierre-Paul, pour répondre à ses attentes, pour obtenir ce que ce dernier n'avait pu obtenir. Pierre-Paul commence par tenter de le raisonner: "Écoute. Je te parle. (p. 80) Tout le contraire du père, donc. Mais, ne réussissant pas à convaincre son frère de changer d'idée, entendez la réplique de Pierre-Paul, où la langue paternelle remonte à la surface, "indice "(dans le sens que Barthes donnait à ce terme) qu'il est en train de perdre la partie: "Maudit câlice! Quoi j'ai fait de mal? Peut pas me faire ça! Tout donné. Maudit. Maudit. Maudit!... L'antonyme est un mot qui, par le sens, s'oppose directement à un autre. Le contraire d'antonyme est synonyme. Le contraire de synonyme est antonyme. L'antonyme est un mot..." (p. 80-81) La seule arme de Pierre-Paul, "sa" langue, vient de s'enrayer; il eût voulu être le contraire des membres de sa famille; il eût voulu être l'antonyme familial... Cependant, les règles de grammaire n'imposeront pas leur loi; elles ne font plus le poids devant les règles familiales. La mise à mort peut désormais avoir lieu; rien ne peut l'empêcher.

Enfin, French Town et sa (mise en) demeure identitaire. Une chose est certaine: Pierre Karch en a eu le goût coupé : $~ \grave{A}$ l'heure du village global et du libre-échange, penser en termes de racines me paraît aussi profond que creux "; "French Town est une pièce du terroir, comme La terre paternelle était un roman du terroir"; et l'estocade finale: * Franco-Ontariens et Franco-Ontariennes, tant que sera primé ce genre de discours, vous ne serez pas sortis du bois. ${ }^{8}$ Excès de surtexte identitaire? J'en ai bien peur. Primo, la dialectique de l'enracinement et du déracinement est beaucoup plus complexe dans Frencb Town que ne le com-

8 Pierre Karch, *French Toum de Michel Oucllette-, Francophonics diAmérique, $n^{0} 5,1995$, p. 92 . 
prendra jamais, semble-t-il, le commentateur; secundo, mutadis mutantis, si French Town est une pièce du terroir, Les bellessceurs seraient une pièce du terroir urbain; tertio, Franco-Ontariens et Franco-Ontariennes, tant que sera publié ce genre de discours critique, vous ne serez pas sortis du bois herméneutique. Serait-ce que l'arbre aurait caché la forêt (ou l'inverse)?

La critique de Pierre Karch atteste ce que j'évoquais en début d'article à propos des corps prescriptifs institutionnels. French Town peut nourrir évidemment un surtexte identitaire (le mépris et la vindicte en moins), mais le dispositif textuel, sa mise à feu et sa déflagration langagière par les personnages le dépassent. French Town ou le surpaysement dépaysant. Arrive un moment où les destins de Cindy, Martin et Pierre-Paul transcendent le passé et ses ombres narrés par la mère. Survient ce point de chute ou de fuite ou d'ancrage ou de rupture où les personnages s'élèvent jusqu'à eux-mêmes, reçoivent la révélation de ce qu'ils veulent être ou de ce qu'ils ne pourront plus être. French Town est un miroir brisé où se regardent des personnages en quête d'eux-mêmes, sans savoir que l'image qu'ils cherchaient n'était pas celle qu'ils allaient trouver.

À miroir brisé, identités spéculaires. Dès lors, je reviens à l'imposture identitaire dans la perspective où, comme le disait Jean Larose, "la littérature défait les identités, elle a même la vertu troublante de révéler l'étrangeté de ce qui nous semble le plus familier" $"$.

\section{Grand ciel bleu par ici: leçons d'existence}

un état voire une province loin loin en deçà de la poésie (p. 8)

Pour clore cet article, j'ai retenu le dernier recueil de poèmes de Robert Dickson, animateur lié de très près au renouveau culturel franco-ontarien des années soixante-dix et quatre-vingt ${ }^{10}$.

9 Jean Larose, "Le fantôme de la littérature., L'amour du pauvre, Montréal, Boréal, coll. - Papiers collés -, 1991, p. 23.

10 - Au cours des années 70-80, une littérature véritablement autochtone en Ontario français a vu ke jour, affirmant haut et fort son caractère distinctif et traitant des questions d'identité, de statut minoritaire, d'errance et de norditude. On trouve associés à ce mouvement les noms d'André Paiement, Jean Marc Dalpé, Robert Dickson, Brigitte Haentjens, Patrice Desbiens, etc.* 
Qu'en est-il avec Grand ciel bleu par ici? En exorde, je laisse pour l'instant au vestiaire le déictique de lieu que les orpailleurs de la franco-ontariennité ne manqueront peut-être pas de brandir comme une marque identitaire revendicatrice, mais qui me contraindrait à chercher ce que je considère comme les véritables enjeux de la poésie de Dickson là où ils ne se trouvent pas. Dans ce recueil, la poésie ne loge pas du côté de la cause; de la revendication, de l'excès, mais plutôt du côté de l'accès: à elle-même, aux amis, à la nature, aux voyages, au cinéma, et surtout à la femme aimée.

Dickson met en scène sa "poétique dans une suite de cinq poèmes sous-titrés : l'air de rien, ce. Citons les deux premiers vers : "l'air de rien ce poème cherche / se cherche une forme, un respir "(p. 43). Élargissons un peu leur portée. La poésie de Dickson est cet air de rien, ce qui constitue sa grandeur, sa vulnérabilité, son chant désacralisé. (Je n'élève pas en dogme cette observation. Vertigineuse création: l'excès peut la tuer; son contraire aussi; il n'y a pas de règle, sauf celle-là. Georges Mounin, l'un des lecteurs de poésie les plus éclairés qui soient, l'a finement démontré dans un livre que tout amateur de poèmes devrait impérativement lire, Avez-vous lu Char?: "Il n'y a pas de technique, pas de mécanique garantie; pour chaque poète la poétique, fût-ce la plus vieille, est à réinventer; et chaque poète épuise, pour les autres et parfois pour soi, sa propre poétique. " ${ }^{11}$ ) Chercher une forme: comment existera le poème? par quel choix esthétique accédera-t-il à lui-même, atteindra-t-il sa plénitude? Une plénitude au demeurant problématique chez Dickson puisqu'elle est toujours entravée, rompue, tendue vers un déséquilibre qui constitue justement son équilibre, vers des dérapages contrôlés qui maintiennent la réalité sur le qui-vive. Le rythme est ainsi toujours heurté, cassé afin qu'il ne s'emballe pas, qu'il " ne coule pas de source ; la poésie qui en résulte est faite de faux départs, d'arrêts brusques, de demi-tours; elle oscille entre l'immédiateté et le recul, le relâchement et le resserrement; elle s'enroule autour de la réalité, la modalise en de multiples fragments d'un dialogisme polymorphe.

Elisabeth Lasserre, "In poète au scuil de l'écriture: l'exiguité selon Patrice Desbiens *, La litténature franco-ontarienne: enjeux estbétiques, op. cit., p. 27.

11 Georges Mounin, Avez-zous lu Char?, précédé de La communication poétique, Gallimard, 1968, p. 137. 
Dans Grand ciel bleu par ici, les mots se répandent à la surface des choses, enserrent la réalité immédiate. Les mots jettent leurs filets sur des lieux et des paysages, sur les sensations et les émois qui en résultent, créant ainsi des agrégats de signification, qui, liés les uns aux autres, dessinent les contours plus ou moins accusés d'un monde, du monde. Tantôt légère, tantôt grave, la poésie va son chemin, s'arrêtant ici, s'amusant là, s'étonnant de l'air et du temps, s'inquiétant de l'air du temps, saisissant à la dérobée la légère sensation de vivre: "dans sa cuisine ce poème sent parfois/qu'il sait quasiment vivre en prend note/le temps de le dire sans plus . (p. 47). Tenant le pari d'une simplicité habitée, de la vie quotidienne traversée par les odeurs, les bruits, les éclats de rire, les pleurs, Dickson impose une poésie qui se refuse à tout emportement métaphorique, à toute surpoétisation qui romprait, par surcharge rhétorique, les instants d'abandon, les moments de frayeur dans la proximité des choses et des êtres. En somme, la poésie de Dickson suit "le cours des choses" (p. 8), est à l'affût de "cette présence grandiose de simplicité/et [du] désir [qui] s'y lit comme un poème qu'on aime/depuis longtemps * (p. 97). Les mots, la simplicité, le désir, le poème, voilà les outils de Dickson; ils en valent bien d'autres, malgré des vers mal rabotés, des images trop faciles, des strophes usées mais qui laissent passer la lumière.

Grand ciel bleu par ici marque une rupture dans une certaine poésie franco-ontarienne. François Paré évoquait la "pratique de la négativité " 12 pour circonscrire les axes sémantiques qui cadastraient quelques-unes des œuvres vives du thêatre et de la poésie. Parmi les poètes, il citait Robert Dickson. Il serait difficile d'annexer Grand ciel bleu par ici à cette pratique. Nous assistons avec ce recueil à un renversement de la négativité. Dickson est passé des Abris nocturnes (titre de son avant-dernier recueil) au Grand ciel bleu par ici, qui atteste une acceptation de l'existence prismatique, sans qu'il soit nécessaire de faire entendre les grandes orgues identitaires. Dickson arrive à ce qui est (à ce qu'il est), à l'affirmation d'une vie qui les vaut toutes et que vaut n'importe quelle (ô Sartre!). Dans ce contexte, le titre marque moins une revendication territoriale, un repliement sur un lieu idyllique franco-ontarien, que la reconnaissance "naturelle" d'un endroit quel qu'il soit - où la douceur de vivre prend corps: ales goé-

12 François Parć, "Pour rompre le discours fondateur: la littérature et la détressè. op. cit., p. 17. 
lands et les enfants sont/à la pêche ça rit et ça crie sous/le grand ciel bleu par icin (p: 60). En outre, il peut même se permettre d'écrire ces vers: *un état voire une province loin/loin en deçà de la poésie" (p. 8). Ce "loin" réitéré, dont on mesure toute la charge (contenue), prend valeur d'exemple: la poésie naît de cette conscience de l'éloignement. Mieux: la poésie sera toujours en avant - en avant de tout, y compris de quelque pays que ce soit. Serait-ce l'ultime illusion identitaire mise à mal? J'ignore comment seront reçus - si tant est qu'ils le soient - et "compris " ces vers. Pour ma part, je les juge à ce point révélateur que j'ose avancer cette alternative: la surcontextualisation identitaire en Ontario français aurait imposé jusqu'à présent son discours sui generis à la poésie de Dickson, ou Robert Dickson vient de remettre à la poésie son eccéité!

\section{Familiarisation et défamiliarisation}

La poésie de Grand ciel bleu par ici multiplie les marques langagières familières. Ce choix esthétique, qui n'a pas à être magnifié au nom d'un supposé et illusoire tutoiement de la réalité, ni décrié à cause d'une fausse désacralisation littéraire, constitue un leurre rhétorique: quelle construction formelle se trahira le moins, trahira moins le sens (pris dans l'absolu) et moins le sens que l'on veut conférer au sens? Le travail langagier n'est pas inclusif, mais exclusif: toutes les formes dont on s'est délesté pour retenir celles qui répondaient le moins imparfaitement au primat esthétique. D'autre part, en Ontario français, la poésie de plusieurs poètes, au premier rang desquels Patrice Desbiens, a transité par la langue parlée familière, pour ne pas dire ordurière, scatologique, tout ensemble happening textuel, saccage symbolique et exorcisme identitaire. Dans cette perspective contextualisante, la poésie familière possède-t-elle des "airs de famille" franco-ontariens? Dans * L'institution littéraire franco-ontarienne et son rapport à la construction identitaire des Franco-Ontariens *, François Paré soutenait que

[c]ontrairement aux fonctions de edéfamiliarisation - que Jonathan Culler (1989) décrit comme essentielles à la littérature, celle-ci doit plutôt engendrer * familiarisation * et collusion. C'est qu'en Ontario français, comme dans toutes les petites cultures dominées, la "défamiliarisation - paraîtrait sans doute trop douloureusement reproduire les conditions d'aliénation dans lesquelles les Franco-Ontariens vivent tous les jours. 
Quelles sont ces caractéristiques de * familiarisation ? J'en retiendrai quatre. Ce sont, d'abord, l'oralité présumée de la littérature franco-ontarienne (à peuple oral, littérature orale!); ensuite, son rejet de la vie intellectuelle et universitaire, en particulier; puis, sa littérarisation de l'espace géographique nordontarien; et, enfin, sa célébration de la marginalité absolue de l'écrivain. ${ }^{13}$

Cette citation appelle plusieurs remarques. Premier paradoxe: ce rejet de la vie intellectuelle et universitaire prit corps au sein même de l'université, en l'occurrence l'Université Laurentienne ${ }^{14}$. Deuxième paradoxe: l'oralité sacralisée et la marginalité absolue de l'écrivain furent relayées par la mise sur pied d'une troupe de théâtre (TNO) et d'une maison d'édition (Prise de Parole); bref, par des structures institutionnelles qui entraient en conflit, de par leur nature même, avec l'oralité, le rejet de la vie intellectuelle et la marginalité de l'écrivain. Ces paradoxes ne sont qu'apparents et se résolvent si on ne perd pas de vue que ces * caractéristiques de familiarisation " ont constitué un corps de prescriptions idéologiques récupéré par des instances légitimantes qui les reconnurent comme telles et les promurent.

Par ailleurs, la problématique des * petites cultures dominées * justifie-t-elle le parti pris de la *familiarisation"? Comment ne pas entendre en écho à cette familiarisation, les propos de Jean Larose :

Pour que la littérature québécoise mérite sa place dans l'éducation québécoise, pour qu'elle en gagne une dans les classes de toute la francophonie, l'institution littéraire doit d'abord lui rendre son essence littéraire, sa dignité fantomatique, cesser de la traiter comme un bien de famille. La littérature ne fait pas partie d'un patrimoine familial. On trompe les étudiants et on trahit la littérature en présentant les œuvres comme des choses à notre image et à notre ressemblance ${ }^{15}$

13 François Paré, * L'institution littéraire franco-ontarienne et son rapport à la construction identitairc des Franco-Ontariens -, dans Jocelyn Létourneau (dir.) avec la collaboration de Roger Bernard, La question identitaire au Canada francopbone. Rëcits, parcours, enjeux, bors-lieux, Sainte-Foy, Presses de l'Université Laval, coll. * Culture française d'Amérique *, p. 53.

14 a Vers 1970, donc, il y a à Sudbury, autour de Robert Dickson et de Firnand Dorais, tous deux nouvellement installés à l'Université Laurentienne, une véritable coalescence des forces 'vives de la jeune culture francophone du Nord. . Ibid., p. 48.

15 Jean Larose, "L.c fantôme de la littérature *, op. cit., p. 24-25. 
L'exhortation de Larose contient, malgré son raccourci épistémologique, une dénonciation d'un groupe de production restreinte qui contrôlerait certains lieux légitimants des champs littéraire et culturel et imposerait une clôture institutionnelle à la littérature québécoise, au-delà de laquelle son ticket ne serait plus valable! Mais, allons à l'essentiel : la familiarisation comme trahison de la littérature (Larose); la familiarisation comme linéament communautaire (Paré). Ces deux pôles antinomiques ne dialectisent-ils pas plusieurs débats qui ont marqué la littérature canadiennefrançaise: des régionalistes aux exotistes, des défenseurs aux détracteurs du joual, du passage de la littérature canadienne-française à la littérature québécoise et, en Ontario français, de la "conscience à l'oubli * ${ }^{16}$, de la * souchitude et de l'orignalitude "17, de la surcontextualisation à la décontextualisation? Qu'il s'agisse de familiarisation (ou de défamiliarisation), nous demeurons toujours dans l'ordre d'un coup de force institutionnel, dans l'ordre d'une imposture fondée puisque légitimée, ce qui la transforme en posture institutionnelle jusqu'à ce qu'elle soit rejetée, remplacée par une autre et qu'elle redevienne une imposture.

Enfin, terminons avec une phrase que j'énonce sur le mode optatif: ce que souhaite Jean Larose pour la littérature québécoise, comment ne pas le souhaiter pour la littérature francoontarienne.

16 François Paré, *Conscience et oubli : les deux misères de la parole francoontarienne , Les littératures de l'exiguitté, Ottawa, Le Nordir, 1992, p. 123-135.

17 Hédi Bouraoui, - Souchitude et orignalitude -, Liaison, $\mathrm{n}^{\circ} 85,15$ janvier 1996 , p. 24-26. 